\title{
Deformation of Nanoporous Materials in the Process of Binary Adsorption: Methane Displacement by Carbon Dioxide from Coal
}

Nicholas J. Corrente ${ }^{1}$, Katarzyna Zarębska ${ }^{2}$, and Alexander V. Neimark ${ }^{1^{*}}$

${ }^{1}$ Department of Chemical and Biochemical Engineering, Rutgers, The State University of New Jersey, Piscataway, NJ 08854, United States

${ }^{2}$ AGH University of Science and Technology, Al. Mickiewicza 30, 30-059, Krakòw, Poland

\section{Supplementary Information:}

\section{Section 1: Estimation of the $\mathrm{CO}_{2}$ fraction in the equilibrium gas phase during adsorption measurements}

The $\mathrm{CO}_{2}$ fraction in the gas phase was only experimentally measured at the highest pressure. To estimate the $\mathrm{CO}_{2}$ fraction during the process of adsorption, we employ the material balances. Taking the mixture composition of feed gas, $y_{\mathrm{CO}_{2}}^{\text {feed }}=y_{f}$. The total amount of gas can be taken as the sum of adsorbed and bulk gas in the apparatus, $N_{t o t}=N_{a d s}+N_{g a s}$. Thus, the ratio of total amount of each gas component can be taken as

$$
N_{\mathrm{CO}_{2}}^{t o t} / N_{C H_{4}}^{t o t}=y_{f} /\left(1-y_{f}\right)(\mathrm{S}
$$

Therefore

$$
\left(1-y_{f}\right) N_{C O_{2}}^{t o t}=y_{f} N_{C H_{4}}^{t o t}
$$

At each measurement, the total amount of species $i$ in the apparatus can be taken as

$$
N_{i}^{\text {tot }}=N_{i}^{a d s}+y_{i} \cdot p V_{g} / R T \text {, }
$$

where $V_{g}$ is the gas volume divided by the mass of coal.

Taking the composition at the highest pressure $p=p^{*}$ as a reference state, $y_{\mathrm{CO}_{2}}\left(p^{*}\right)=y^{*}$. The total amount of adsorbed gas at this reference state for each component is then

$$
N_{C O_{2}}^{a}=\frac{N_{1}^{0} K_{1} y^{*} p^{*}}{1+K_{1} y^{*} p^{*}+K_{2}\left(1-y^{*}\right) p^{*}} ; N_{C H_{4}}^{a}=\frac{N_{2}^{0} K_{2}\left(1-y^{*}\right) p^{*}}{1+K_{1} y^{*} p^{*}+K_{2}\left(1-y^{*}\right) p^{*}}
$$

Combining Equations S2, S3, and S4 yields,

$$
\left(1-y_{f}\right) N_{C O_{2}}^{a}\left(y^{*}, p^{*}\right)-y_{f} N_{C H_{4}}^{a}\left(y^{*}, p^{*}\right)=-\left[\left(1-y_{f}\right) y^{*}-y_{f}\left(1-y^{*}\right)\right] p^{*} V_{g} / R T, \text { (S5) }
$$

For measurements at $p=p^{*}$, the gas volume can be determined from

$$
V_{g}=\frac{\left(1-y_{f}\right) N_{C O_{2}}^{a}\left(y^{*}, p^{*}\right)-y_{f} N_{C H_{4}}^{a}\left(y^{*}, p^{*}\right)}{\left(y_{f}-y^{*}\right) p^{*} / R T}
$$

For each pressure point, $y_{\mathrm{CO}_{2}}$ can be estimated from the algebraic equation 


$$
\left(1-y_{f}\right) N_{C O_{2}}^{a}\left(y_{C O_{2}}, p\right)-y_{f} N_{C H_{4}}^{a}\left(y_{C O_{2}}, p\right)=\left(y_{f}-y_{C O_{2}}\right) p V_{g} / R T,(\mathrm{~S} 7)
$$

Combining Equations S6 and S7 then yields

$$
\frac{\left(1-y_{f}\right) N_{C O_{2}}^{a}\left(y_{C_{2}}, p\right)-y_{f} N_{C H_{4}}^{a}\left(y_{C_{2}}, p\right)}{\left(y_{f}-y_{C O_{2}}\right) p / R T}=\frac{\left(1-y_{f}\right) N_{C O_{2}}^{a}\left(y^{*}, p^{*}\right)-y_{f} N_{C H_{4}}^{a}\left(y^{*}, p^{*}\right)}{\left(y_{f}-y^{*}\right) p^{*} / R T} \text {. (S8) }
$$

Substituting Equation 5 for $N_{i}^{a}$ in Equation S8 yields the following system of equations, which can be solved to find $y=y(p)$

$$
\begin{gathered}
f(y, p)=\frac{\left(1-y_{f}\right) y N_{1}^{0} K_{1}-y_{f}(1-y) N_{2}^{0} K_{2}}{\left(y_{f}-y\right)\left(1+\left(y\left(K_{1}-K_{2}\right)+K_{2}\right) p\right)} \\
f\left(y^{*}, p^{*}\right)=\frac{\left(1-y_{f}\right) y^{*} N_{1}^{0} K_{1}-y_{f}\left(1-y^{*}\right) N_{2}^{0} K_{2}}{\left(y_{f}-y^{*}\right)\left(1+\left(y^{*}\left(K_{1}-K_{2}\right)+K_{2}\right) p^{*}\right)},(\mathrm{S} 9)
\end{gathered}
$$




\section{Section 2: Separate contributions of the strain of pure $\mathrm{CH}_{4}$ and $\mathrm{CO}_{2}$ at geological conditions}

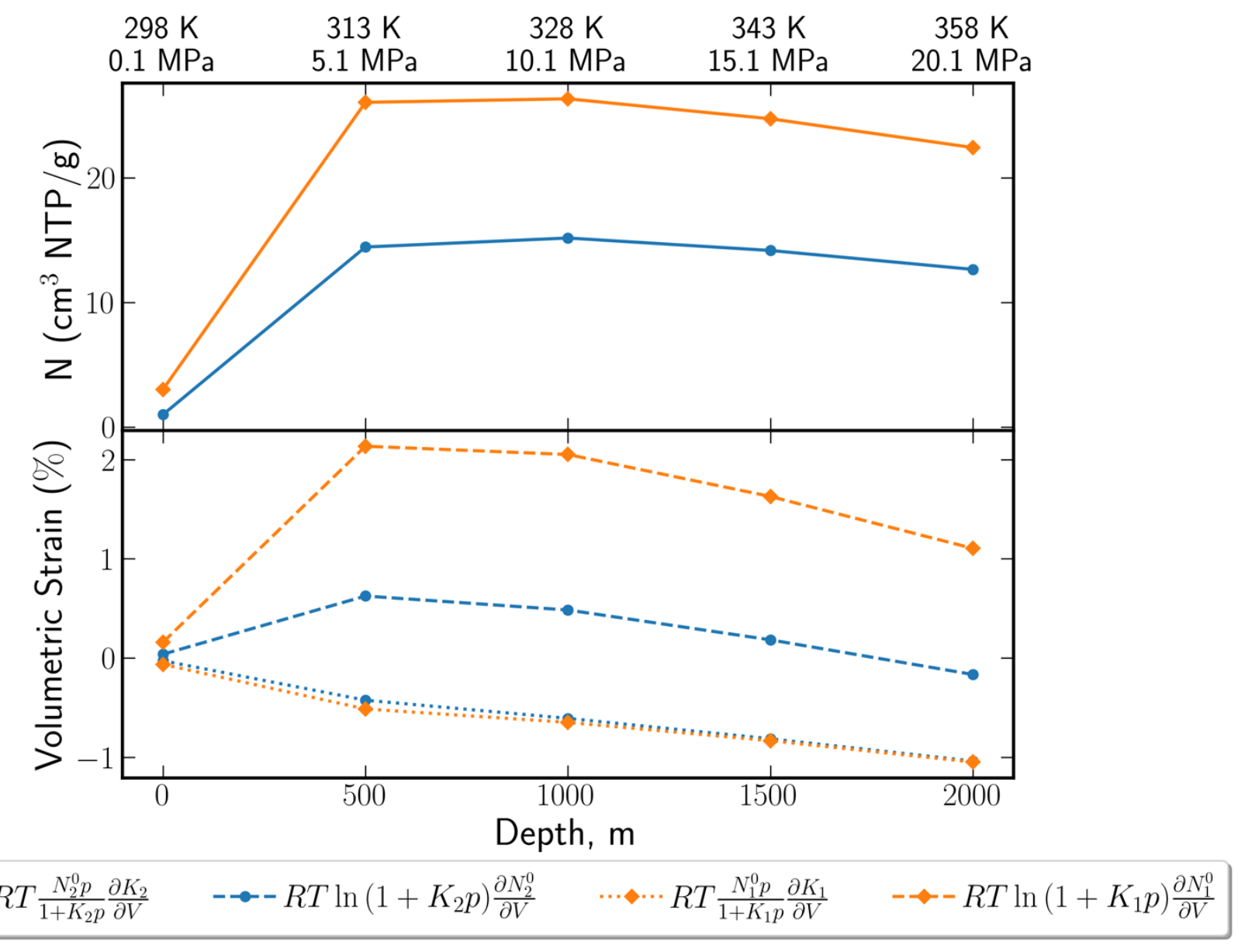

Figure S1: Pure component adsorption and strain dependencies on the depth of the reservoir presented in Figure 2. $\mathrm{CH}_{4}$ data is represented in blue and $\mathrm{CO}_{2}$ data in orange. Strain in the lower panel is decomposed into the two competing terms in Equation 10. The dotted and dashed lines show respectively the contributions proportional to the Henry constant susceptibility factor, $\lambda_{K_{1 / 2}}$, and the adsorption capacity susceptibility factor, $\lambda_{N_{1 / 2}^{0}}$.

Figure S1 shows the pure component adsorption and strain dependencies on the reservoir depth due to the respective increase of pressure and temperature, pictured in Figure 2, with the strain dependence (lower panel) decomposed with respect to two terms in Equation 10. The nonmonotonic behavior of the adsorption and strain are attributed to the competing temperature and pressure affects. With increasing pressure, the positive adsorption capacity susceptibility factor, $\lambda_{N_{1 / 2}^{0}}$, yields swelling (increase in the strain over the surface level conditions), as shown by dashed lines, while the negative Henry constant susceptibility factor, $\lambda_{K_{1 / 2}}$, yields contraction. As the reservoir depth increases, the 
temperature effects dominate due to the progressively decreasing Henry constant according to Equation 6.

\section{Section 3. Correlation of theoretical predictions and experimental data for binary adsorption and strain isotherms on sample B32.1.}

Figure S2 shows the theoretical predictions from Equations 4, 8, and 9 compared with the experimental adsorption and strain measurements for pure $\mathrm{CO}_{2}$ and $\mathrm{CH}_{4}$, as well as a mixture with $\mathrm{CO}_{2}$ fraction $y_{\mathrm{CO} 2}=0.234$, on coal sample B32.1. Figure S2 shows very similar trends as in Figure 1: the model is able to predict the mixture adsorption and strain. Note a slight decrease of the pure $\mathrm{CH}_{4}$ strain isotherm at low pressures, suggesting the contraction of the sample, as discussed in Section 3.1 of the main text. Model parameters for sample B32.1 are presented in Table S1.
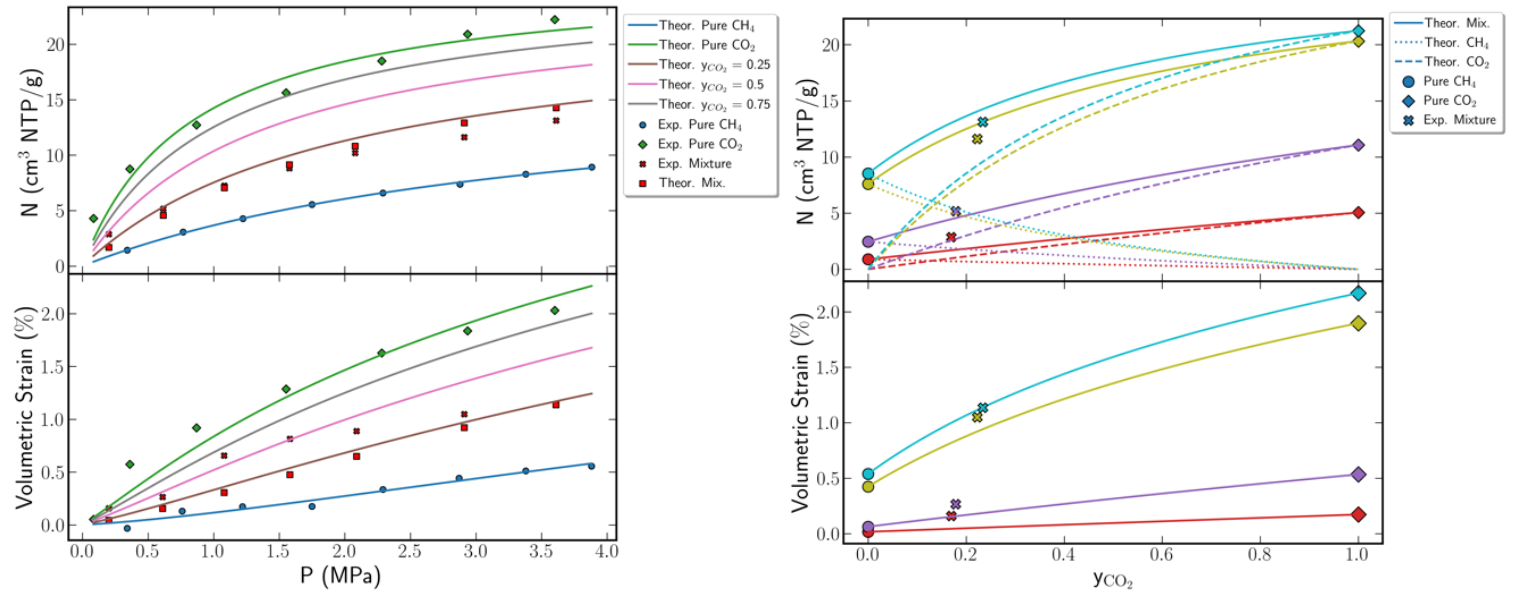

Figure S2: Left: Adsorption (upper) and strain (lower) isotherms for pure CO2 (green), pure $\mathrm{CH}_{4}$ (blue), and binary mixtures (red, other colors) on sample B32.1. Green points represent experimental pure $\mathrm{CO}_{2}$ data, blue points represent pure $\mathrm{CH}_{4}$ data, red crosses represent experimental mixture data, lines and red squares represent pure species and mixture predictions, respectively from Eqs. (5) and (8). Right: Prediction of the variation of adsorption and strain as functions of the $\mathrm{CO}_{2}$ fraction, ycO2, in the process of displacement of $\mathrm{CH}_{4}$ by $\mathrm{CO}_{2}$ at various gas pressures. Experimental data points correspond to pure $\mathrm{CO}_{2}$ (diamonds) and $\mathrm{CH}_{4}$ (circles) at yco2 $=0,1$, and mixture (crosses) at the $\mathrm{CO}_{2}$ fraction estimated from material balances. Predictions for the mixture (solid lines) and pure $\mathrm{CO}_{2}$ and $\mathrm{CH}_{4}$ (dashed and dotted lines, respectively) are calculated at pressures of 0.201 MPa (red), 0.615 MPa (purple), 2.911 MPa (yellow), and 3.611 $\mathrm{MPa}$ (cyan). The strain is counted from the reference "dry" state of the adsorbent at $\mathrm{P}=0$.

Table S1: Model parameters for sample B32.1 determined from the pure component adsorption and strain isotherms. 


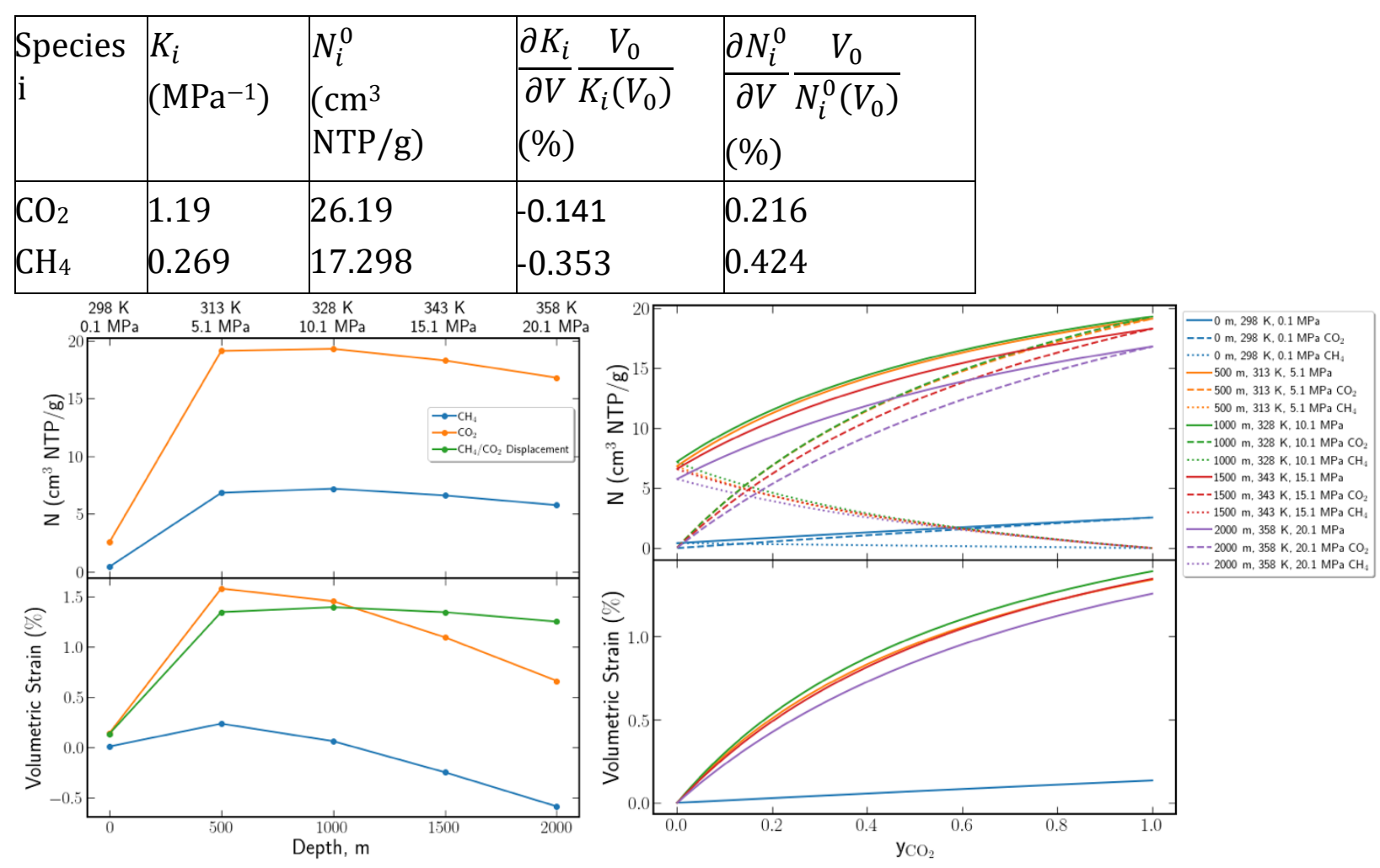

Figure S3: Prediction of adsorption and deformation for sample B32.1 upon the displacement of $\mathrm{CH}_{4}$ by $\mathrm{CO}_{2}$ at geological conditions at the levels of $0,500,1,000,1,500$, and 2,000 $\mathrm{m}$ below the surface. Left: Adsorption and strain of the coal sample with pure $\mathrm{CH}_{4}$ and $\mathrm{CO}_{2}$ at geological conditions. The strain is counted from the reference state of the "dry" adsorbent at $T=298 \mathrm{~K}$ and $\mathrm{P}=0$. The strain difference due to the complete displacement of $\mathrm{CH}_{4}$ by $\mathrm{CO}_{2}$ is show in green. Right: Adsorption (upper) and strain (lower) isotherms as a function of bulk $\mathrm{CO}_{2}$ fraction at yco2 and the respective pressure and temperature shown in the figure caption. Solid, dotted, and dashed lines represent respectively the total adsorption and strain, $\mathrm{CH}_{4}$ and $\mathrm{CO}_{2}$ adsorption. The strain is counted from the state of the adsorbent saturated by pure $\mathrm{CH}_{4}$ at given pressure.

Figure S3 shows the theoretical adsorption and strain predictions for sample B32.1, similar to the predictions for sample B32.2 shown in Figure 2 of the main text. Sample B32.1 exhibits comparable predications to B32.2 with increasing reservoir depth and the respective increase in temperature and pressure. Adsorption and swelling increase significantly from surface level conditions up to $500 \mathrm{~m}$, after which the adsorption plateaus at approximate $17 \mathrm{~cm}^{3} \mathrm{NTP} / \mathrm{g}$ and $6 \mathrm{~cm}^{3} \mathrm{NTP} / \mathrm{g}_{\text {for }} \mathrm{CO}_{2}$ and $\mathrm{CH}_{4}$, respectively. Below depths of $500 \mathrm{~m}$, the pure component strain decreases significantly as a result of the dominating temperature affects. The strain during displacement of $\mathrm{CH}_{4}$ by $\mathrm{CO}_{2}$ plateaus at approximately $1.3 \%$. 